\title{
Geomagnetic activity and wind velocity during the Maunder minimum
}

\author{
B. Mendoza \\ Instituto de Geofísica UNAM, Circuito Exterior Ciudad Universitaria, 04510 México DF, México \\ Received: 20 June 1996 / Revised: 2 December 1996 / Accepted: 3 December 1996
}

\begin{abstract}
Following a given classification of geomagnetic activity, we obtained $a a$ index values for the Maunder minimum (1645-1715). It is found that the recurrent and fluctuating activities were not appreciable and that the shock activity levels were very low. The $a a$ index level was due almost entirely to the quiet days. Calculated average solar-wind velocities were 194.3 $\mathrm{km} \mathrm{s}^{-1}$ from 1657 to 1700 and $218.7 \mathrm{~km} \mathrm{~s}^{-1}$ from 1700 onwards. Also, the coronal magnetic field magnitude and southward interplanetary magnetic field component $B z$ were lower. It is concluded that the nearly absent levels of geomagnetic activity during this period were due to lower coronal and $B z$ magnetic field magnitudes as well as to the continuous impinging on the Earth of a slow wind.
\end{abstract}

\section{Introduction}

The Maunder minimum (1645-1715) is now recognized as a period of depressed solar activity, signaled by a reduction in naked-eye sunspot number, diminished corona at eclipses, suppressed auroral incidence, and marked increase in the radiocarbon production detected in tree rings (Eddy, 1980).

Sunspots are generated by toroidal magnetic fields lying below the photosphere brought to the surface by buoyancy. For example, the $\alpha-\omega$ dynamo theory (Krause and Radler, 1980) indicates that the stronger the star's rotation rate the stronger the deformation of the poloidal field component below the star's surface and the generation of the toroidal component. If for some reason the Sun's rotation rate was lower during the Maunder minimum than at present, the toroidal field may have been weaker and the production of sunspots lower than today. The toroidal field derives from the poloidal field of the previous half solar cycle and vice versa. Poloidal fields are large scale and extend into the corona. It is now widely accepted that the heating of the star's atmosphere is magnetic (Axford and McKenzie, 1992; Habbal, 1992). A weaker field produces less heating of the Sun's atmosphere and then lower temperatures. Also, emission lines like the $\mathrm{Ca}$ II $\mathrm{H}$ and $\mathrm{K}$ lines that brighten with increasing magnetism (Leighton, 1959; Howard, 1959) would be dimmer. Lower coronal temperatures produce a slower solar wind (Parker, 1963). Further, the mean value of the southward component $B z$ of the interplanetary magnetic field is proportional to the sunspot number (Slavin and Smith, 1983). As the Maunder-minimum period was characterized by low sunspot numbers, we can conclude that the solar wind in this epoch was slower and smoother than at present and therefore produced an inefficient magnetic reconnection that in turn resulted in a low production of aurorae.

Suess (1979) has proposed qualitative arguments suggesting that during the Maunder minimum the solar magnetic field, the solar mass flux, and the velocity and density of the solar wind were reduced, and also that the interplanetary magnetic field was smooth. Based on the results of models of the quiet solar wind developed by several authors, Suess proposed that the wind speed at the Earth's orbit was $\leq 300 \mathrm{~km} \mathrm{~s}^{-1}$ and that the density was $\leq 5 \mathrm{~cm}^{-3}$. Later, Silverman (1986) calculated the solar-wind velocity at $1 \mathrm{AU}$ and the values of the $a a$ indices during several solar minima. In particular, for the Maunder-minimum period he concluded that the coronal holes continued sending strong wind streams with velocities $\geq 300 \mathrm{~km} \mathrm{~s}^{-1}$, and that the major factor in the decline of geomagnetic and therefore auroral activities was not the solar-wind velocity but the reduction of the $B z$ component of the interplanetary magnetic field.

It is still highly controversial whether during the Maunder minimum there was an $\sim 11$-year cycle or not. Kocharov (1987) has shown the variations of ${ }^{14} \mathrm{C}$ with time from 1600-1730, finding that they had a cycle closer to 22 years. Silverman (1992) indicates that 
during the Maunder minimum the 11-year cycle disappears in the frequency of auroral occurrence. On the other hand, Ribes et al. (1989) show that during this minimum the horizontal solar diameter had variations dominated by a 9.4-year periodicity. Schroeder (1992) demonstrates that the 11-year cycle in solar and auroral activity existed during this period. Ribes and NesmeRibes (1993) identify an approximately 11 -year cycle in sunspot numbers.

In the present work we assume the existence of an 11year cycle in the sunspot numbers during the Maunder minimum. Also, for this period we adopt the cycle lengths suggested by Ribes and Nesme-Ribes (1993), taken from minimum to minimum: $1646-1657 ; 1658-1668 ; 1669$ $1679 ; 1680-1690 ; 1691-1700 ; 1701-1710 ; 1711-1721$. We took 1723 as the last year of the last cycle instead of 1721 , in order to match the Wolf numbers of the cycles plotted by Hoyt et al. (1994) after 1700.

In our study we shall apply a number of equations which hold for modern times; we should point out that using these relations for the Maunder minimum is inherently speculative as the Sun during this period might have behaved quite differently.

The paper is organized as follows: in Sect. 2 we obtain the values of the shock, recurrent, and fluctuating activities; in Sect. 3 the quiet days are found; Sect. 4 has the calculated solar-wind velocities; Sect. 5 presents the estimations of the magnetic field, and in Sect. 6 we present the discussion and conclusions.

\section{The geomagnetic activity during the Maunder minimum}

In recent years Legrand and Simon (1985) and Simon and Legrand $(1986,1987)$ have classified the geomagnetic phenomena according to their interplanetary sources for the period 1868-1986. They relate the quiet days to the flow of low-speed winds coming from the heliospheric neutral current sheet. They have also distinguished three classes of geomagnetic activity: the shock activity due to traveling interplanetary shock waves, the recurrent activity, produced by high-speed wind streams coming from polar coronal holes, and the fluctuating activity originated by moderate-speed wind associated with the turbulence in the heliospheric current sheet. More recently Pérez-Enríquez and Mendoza (1993) showed that the fluctuating activity is specifically related with the Earth's sector boundary crossings.

Under the proposed scheme the annual $a a$ index represents the sum of the quiet days plus the shock, recurrent, and fluctuating activities. Its value is given by the ratio of the annual sum of daily indices and the annual number of the related days.

Legrand and Simon (1985) found a relationship between $R z$, the annual average sunspot number of the cycle in progress, and the annual level of the shock geomagnetic activity $a a_{s h}$ :

$a a_{s h}=0.048 R z$.
Although the dispersion of Eq. (1) is large for high sunspot numbers, when the sunspot number is less than $\sim 50$ the scattering is reduced and the relationship between sunspots and shock activity is reasonably well represented by this expression.

Concerning the recurrent geomagnetic activity, Simon and Legrand (1986) found a close relationship between the sum $a a_{r}$ of the recurrent activity during an interval of 4 years around the minimum and the following cycle's sunspot maximum number. They found that no appreciable activity is observed before cycles with solar maximum sunspot numbers less than $\sim 50$, as the following expression indicates:

$a a_{r}=\left(R_{\max }-50\right) / 0.0087$.

Here $R_{\max }$ is the maximum sunspot number of the following cycle.

The annual fluctuating activity occurring at sunspot minimum or on the following year, $a a_{f}$, also presents a good association with the sunspot maximum number of the following cycle. The relationship is given by (Legrand and Simon 1987):

$a a_{f}=\left(R_{\max }-52\right) / 13.13$.

Again, no appreciable fluctuating activity is observed before cycles with maximum sunspot numbers below $\sim 50$.

In Fig. 1 and Table 1 we notice that during the Maunder minimum the highest sunspot number occurred in 1717 and was 4.08 (after Ribes and NesmeRibes, 1993). Therefore, according to the earlier discussion neither the recurrent nor the fluctuating geomagnetic activities were appreciable. The only activity probably sometimes present was the shock activity. In Fig. 2 we plotted the seven points corresponding to the years of the minima during the Maunder minimum. We observe that only in the solar-minimum years of 1710 and 1723 was there shock-wave activity (dashed line), and that its level was very low compared with contemporary values of this kind of activity which reach an average of $\sim 5 \gamma$ (Simon and Legrand, 1987).

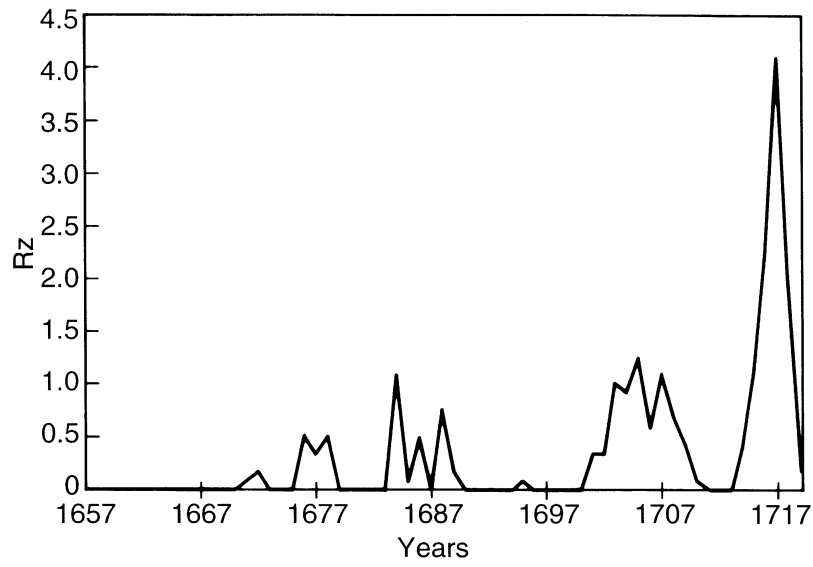

Fig. 1. Annual sunspot number during the Maunder minimum, 16451715, after Ribes and Nesme-Ribes (1993) 
Table 1. Annual sunspot number

\begin{tabular}{|c|c|c|c|}
\hline Year & Sunspot number & Year & Sunspot number \\
\hline 1657 & 0 & 1693 & 0 \\
\hline 1658 & 0 & 1694 & 0 \\
\hline 1659 & 0 & 1695 & 0.08 \\
\hline 1660 & 0 & 1696 & 0 \\
\hline 1661 & 0 & 1697 & 0 \\
\hline 1662 & 1 & 1698 & 0 \\
\hline 1663 & 0 & 1699 & 0 \\
\hline 1664 & 0 & 1700 & 0 \\
\hline 1665 & 0 & 1701 & 0.33 \\
\hline 1666 & 0 & 1702 & 0.33 \\
\hline 1667 & 0 & 1703 & 1 \\
\hline 1668 & 0 & 1704 & 0.92 \\
\hline 1669 & 0 & 1705 & 1.25 \\
\hline 1670 & 0 & 1706 & 0.58 \\
\hline 1671 & 0.08 & 1707 & 1.08 \\
\hline 1672 & 0.17 & 1708 & 0.67 \\
\hline 1673 & 0 & 1709 & 0.42 \\
\hline 1674 & 0 & 1710 & 0.08 \\
\hline 1675 & 0 & 1711 & 0 \\
\hline 1676 & 0.5 & 1712 & 0 \\
\hline 1677 & 0.33 & 1713 & 0 \\
\hline 1678 & 0.5 & 1714 & 0.42 \\
\hline 1679 & 0 & 1715 & 1.17 \\
\hline 1680 & 0 & 1716 & 2.33 \\
\hline 1681 & 0 & 1717 & 4.08 \\
\hline 1682 & 0 & 1718 & 1.83 \\
\hline 1683 & 0 & 1719 & 0.17 \\
\hline 1684 & 1.08 & 1723 & 0.92 \\
\hline 1685 & 0.08 & & \\
\hline 1686 & 0.5 & & \\
\hline 1687 & 0 & & \\
\hline 1688 & 0.75 & & \\
\hline 1689 & 0.17 & & \\
\hline 1690 & 0 & & \\
\hline 1691 & 0 & & \\
\hline 1692 & 0 & & \\
\hline
\end{tabular}

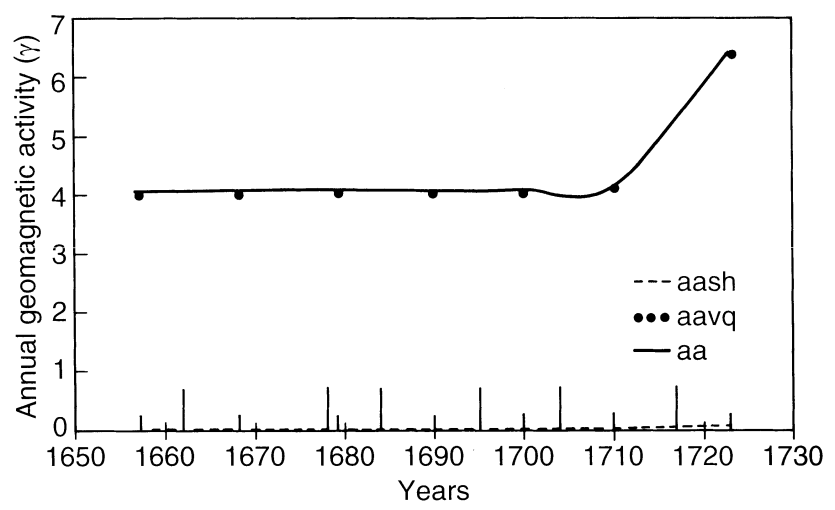

Fig. 2. Solar-minimum annual average index of very quiet geomagnetic times during 1645-1715: $a a_{v q}=4.06+0.019 R_{\max }(r=0.83)$, dots. Solar-minimum annual average index of shock-related geomagnetic activity during 1645-1715 $a a_{s h}=0.048 \mathrm{Rz}$, dashed line. Solarminimum annual average index of geomagnetic activity during 1645 1715 , given by $a a=a a_{v q}+a a_{s h}$, solid line. The thin short vertical lines on the $x$ axis correspond to the years of the solar maximum. The thick ones correspond to the years of the solar minimum

\section{The quiet days}

Quiet days are defined as those with an annual $a a$ index less than $20 \gamma$. Analyzing the quiet days from 1868 to 1986, Simon and Legrand (1987) have concluded that:

1) The annual number of quiet days during the year of the solar minimum is inversely proportional to $R_{\max }$, the maximum sunspot number of the following solar cycle.

2) Also, the higher the annual number of quiet days during the year of the minimum the weaker the annual average $a a_{q}$ index.

Within the quiet-day set there is the very-quiet-day group: those days with $a a<10 \gamma$. Presumably this group corresponds to interplanetary conditions in which the current sheet is very thick, implying weak magnetic fields in the Sun (Simon and Legrand, 1989). Those conditions were probably present during the Maunder minimum. Therefore, our interest will be focused on the very-quiet-day behavior. We analyzed the very-quiet-day data from 1868 to 1986 (Legrand, private communication) and this subset also follows the pattern described in (1) and (2) just given, as can be seen from Fig. 3a, b. Furthermore, we obtain a relationship between the very-quiet-day $a a_{v q}$ and $R_{\max }$. This is shown in Fig. $3 \mathrm{c}$ and the relation found is given by:

$a a_{v q}=4.06+0.019 R_{\max }$.

For this equation the correlation coefficient $r$ is 0.83 .

Knowing the annual sunspot numbers during the Maunder minimum period and using Eq. (4) we obtain $a a_{v q}$ for the years of the solar minima (dots in Fig. 2). Furthermore with the help of Eq. (1) we can calculate $a a$ for the solar minimum years (solid line in Fig. 2) as follows:

$a a=a a_{v q}+a a_{s h}$.

From this figure we notice that the main contribution to the $a a$ index level comes from the quiet days. For the solar minimum years from 1657 to 1700 the average geomagnetic activity level was $4.07 \gamma$. For the solar minimum years from 1710 onwards the activity increased slightly to reach an average level of $5.05 \gamma$.

The number of quiet days is proportional to the thickness of the neutral sheet (Simon and Legrand, 1989). During the Maunder minimum $R z<50$. Therefore, according to Fig. 3a there should have been very quiet geomagnetic days for more than 300 days each year of the period, implying a very thick neutral sheet. For instance, during the minimum of 1878, precursor of one of the lowest maxima of the 19th century $\left(R_{\max }\right.$ $\sim 64$ ), the thickness of the neutral sheet was larger than $14.4^{\circ}$ and the Earth was immersed in a stream of slow wind for most of the year, resulting in a high number of quiet days (Simon and Legrand, 1989). 

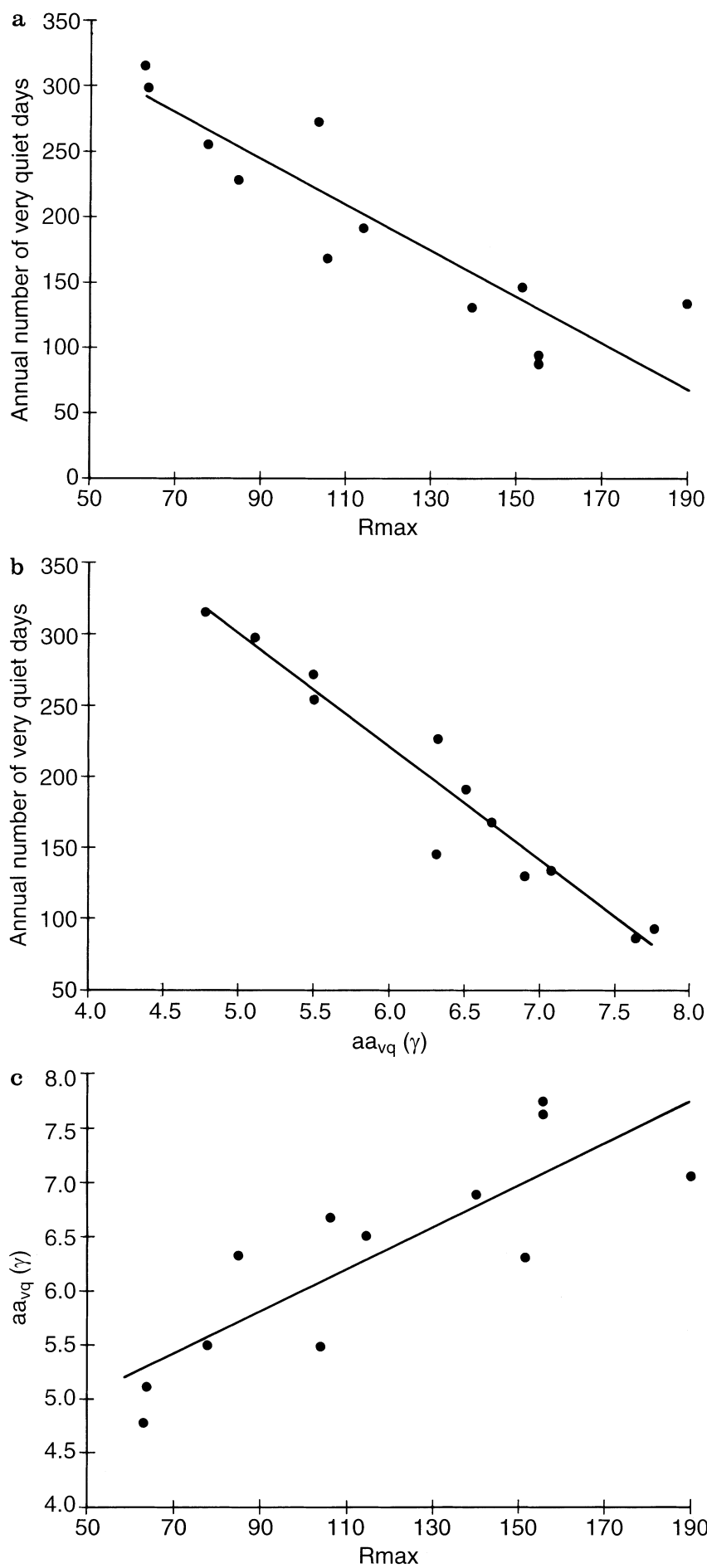

Fig. 3. a Annual number $N$ of very quiet days $(a a<10 \gamma)$ versus the maximum sunspot number $R_{\max }$ of the following solar cycle: $N=-1.697 R_{\max }+392.56, r=0.88$. b Annual number of very quiet days during the year of the minimum versus the annual average $a a_{v q}$ index: $N=-79.12 a a_{q}+6.951, r=0.96$. c Annual average $a a_{v q}$ index versus $R_{\max }: a a_{v q}=4.06+0.019 R_{\max }, r=0.83$

\section{The solar-wind velocity at the Earth's orbit}

In order to calculate the solar-wind velocity at $1 \mathrm{AU}$, the only parameters we know are the annual sunspot number $R z$ from 1666 to 1720 and the values of $a a$ for

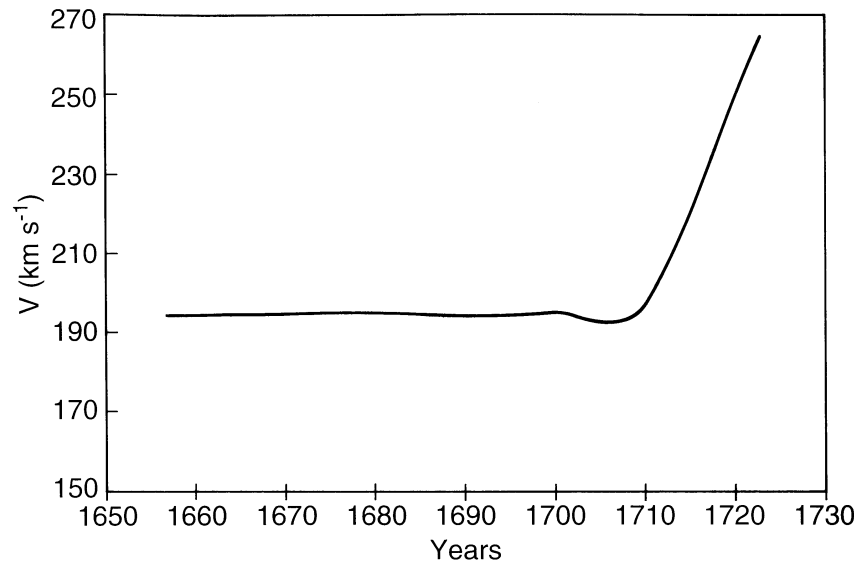

Fig. 4. Solar-wind velocity at Earth's orbit during the Maunder minimum obtained from Eq. (6). The values correspond to the 7 years of solar minima, occurring during this period

the solar-minimum years during the period of interest (Eq. 5).

Feynman and Crooker (1978) have proposed a relationship between $a a$, the velocity of the wind $V$, and the yearly mean $B z$ component (measured in the GSM coordinate system):

$a a=1.3+4.7 \times 10^{-5} B z V^{2}$.

For this expression $r=0.9$.

On the other hand Slavin and Smith (1983) have determined a relationship between the annual mean value of $B z$ (measured in the GSE coordinate system) and the sunspot number given by:

$B z=1.56+7.19 \times 10^{-3} R z$.

For this equation $r=0.93$.

Taking Eqs. (6) and (7) and solving for $V$ we find:

$V=\left\{(a a-1.3) /\left(7.33 \times 10^{-5}+3.38 \times 10^{-7} R z\right)\right\}^{1 / 2}$

In Fig. 4 we observe the results of the calculations. For the solar minimum years from 1657 to 1690 the average wind velocity was $194.3 \mathrm{~km} \mathrm{~s}^{-1}$. For the minimum years $1700-1723$ the average velocity increased slightly, reaching a value of $218.7 \mathrm{~km} \mathrm{~s}^{-1}$.

\section{The magnetic field}

During the year the heliolatitude of the Earth oscillates from $7^{\circ}$ north in September to $7^{\circ}$ south in March, covering a total of around $14^{\circ}$. Also during solar minimum times the current sheet lies in the equatorial plane and its warping is very low (Hoeksema and Scherrer, 1986). Therefore during these periods the Earth is frequently inside the neutral sheet. At the neutral sheet the wind speed falls off and reaches a minimum (Bruno et al., 1986; Kojima and Kakinuma, 1990). Moreover, the thickness of the slow-wind sheet depends upon the intensity of the solar dipole. Evidence that the magnetic field intensity during the Maunder 
minimum was in fact lower than the present one comes from investigations on the behavior of surface magnetic fields in solar-type stars. These investigations have shown that emission in the chromospheric $\mathrm{Ca}$ II $\mathrm{H}$ and $\mathrm{K}$ lines $(\mathrm{HK})$ is a surrogate for magnetic flux (Leighton, 1959; Howard, 1959), i. e.:

$B=B_{0} H K / H K_{0}$

Here the subscript 0 corresponds to contemporary values. The usual $\mathrm{Ca}$ II $\mathrm{H}$ and $\mathrm{K}$ flux ratio observed for solar minimum times is $H K_{0}=0.164$ (Wilson, 1978). It has been shown that the probable $H K$ flux for Maunder-minimum times was between 0.121 and 0.161 for 1683 , when the solar activity was very depressed, and between 0.122 and 0.163 for 1715 when the Sun regained strength (Mendoza, 1996). The lower limits are consistent with the minimum $H K$ flux observed for solar-type stars that are in a noncycling state, the upper limits correspond to cycling stars (Baliunas and Jastrow, 1990). Using Eq. (9) we find values of $B_{c h}$ $=0.74-0.98 B_{\text {cho }}$ for the chromospheric magnetic field during 1683 and of $B_{c h}=0.74-0.99 B_{c h o}$ for 1715 . The chromospheric fields $B_{c h}$ extend up into the corona, producing weaker coronal fields. With a reduced coronal dipole intensity we expect that the Earth should have been immersed inside an even thicker sheet than the one occurring, for instance, in 1878, and therefore received a slow wind for more than 300 days during the year.

Following Eq. (7) we observe that $B z=1.56 \gamma$ for those years in which $R z=0$. From 1658 to 1700 we find an average of $B z=1.57 \gamma$ and from 1701 to 1720 the average is $B z=1.63 \gamma$.

\section{Discussion and conclusions}

We have analyzed the geomagnetic activity level during the Maunder minimum following the classification of the geomagnetic activity given by Legrand and Simon (1985) and Simon and Legrand (1986, 1987). We found that the recurrent and fluctuating activities were not appreciable, and that the shock activity presented very low levels compared with modern average values. The level of the $a a$ index was due almost entirely to the quiet days. The average values found are $\sim 4.1 \gamma$ from 1657 to 1700 and $\sim 5.1 \gamma$ from 1701 to 1723 ; modern values for $a a$ are $\leq 20 \gamma$ (Simon and Legrand, 1989), at most 5 and 4 times larger, respectively, than those for the Maunder minimum. In this sense the geomagnetic activity was nearly absent during the Maunder minimum and only quiet magnetic days existed.

The auroral activity is produced mainly by a combination of shock and recurrent activities (Legrand and Simon, 1988). The nearly absent levels of recurrent geomagnetic activity and the very low levels of shock activity imply depressed auroral activity during the Maunder minimum. In modern times, aurorae related to shock geomagnetic activity appears centered on the invariant latitude of $59^{\circ}-60^{\circ}$ and has an unlimited extension towards the equator (Legrand and Simon, 1989). Taking this behavior as a guide, the aurorae during the Maunder minimum, when they occurred, should have been present at middle latitudes, as has been found (Silverman, 1992).

On calculating the solar-wind velocity at the Earth's orbit, we found that during the solar minimum years from 1657 to 1700 the wind had an average velocity of around $194.3 \mathrm{~km} \mathrm{~s}^{-1}$ and from 1700 onwards this velocity increased, reaching an average value of 218.7 $\mathrm{km} \mathrm{s}^{-1}$. Modern slow solar-wind velocities are very seldom below $250 \mathrm{~km} \mathrm{~s}^{-1}$ (Kojima and Kakinuma, 1990). This value is around 1.3 and 1.1 times higher than the calculated average Maunder-minimum wind velocities.

When we apply Eq. (7) to the years 1966-1982, the period for which this relation holds, an average $B z$ field of around $2.14 \gamma$ is obtained. Maunder-minimum average $B z$ fields of $1.57 \gamma$ from 1658 to 1700 and of $1.63 \gamma$ from 1701 to 1720 are found. Therefore, the modern average $B z$ is around 1.4 and 1.3 times higher than the Maunder $B z$ field. Finally, lower chromospheric $B_{c h M m}=0.7-1 B_{c h o}$, and therefore lower coronal magnetic fields are found which produce a lower heating of the atmosphere, a slower wind leaving the Sun, and a thicker heliospheric current sheet.

We conclude that the low levels of geomagnetic activity and therefore of auroral activity had two causes: a smoother interplanetary magnetic field and the continuous impact on the Earth's environment of slower wind streams as compared to modern times. The wind impinging on the Earth was slower for the following reasons: a) there was a slower wind leaving the corona due to the lower coronal temperature produced by a lower solar magnetic field, and b), since the number of quiet days is proportional to the slow-wind neutral sheet thickness and during the Maunder minimum there were more than 300 very quiet days for most years of this period, the Earth spent almost all the time inside this slow-wind sheet.

We envisage the Maunder-minimum low-speed wind coming out from a region that encompassed coronal helmet streamers and the boundaries of coronal holes, and traveling along the heliospheric current sheet. As this current sheet was very thick and with very low warping the Earth should have been mostly inside the central parts receiving this very low speed solar wind. We also imply that the wind during the Maunder minimum was essentially the same as at the present.

Furthermore, our results suggest that from 1700 onwards the solar activity resumed, but this was not evidenced in sunspots until the following maximum.

Finally, it is important to point out that more recent calculations of sunspot numbers during the Maunder minimum (Hoyt et al., 1994) show even lower numbers than the ones used in the present paper. If these preliminary estimations are in fact more accurate, then the conclusions of the paper shall be reinforced, because the wind velocity and the $B z$ field shall have even lower values. 
Acknowlegements. Topical Editor R. Schwenn thanks S.T. Suess and another referee for their help in evaluating this paper.

\section{References}

Axford, W. I., and J. F. McKenzie, The origin of high-speed solar wind streams, in Solar Wind Seven, Eds. E. Marsh, R. Schwenn, Pergamon Press, Oxford, New York, Seoul, Tokyo, pp. 1-5, 1992.

Baliunas, S. L., and R. Jastrow, Evidence for long-term brightness change of solar-type stars, Nature, 348, 520-523, 1990.

Bruno, R., U. Villante, B. Bassano, R. Schwenn, and F. Mariani, In situ observations of the latitudinal gradients of the solar wind parameters during 1976, 1977, Solar Phys., 104, 431-445, 1986.

Eddy, J. A., The historical record of solar activity, in The Ancient Sun: Fossil Record in the Earth, Moon and Meteorites, Eds. R. O. Pepin, J. A. Eddy, R. B. Merrill, Pergamon Press, New York, pp. 119-134, 1980.

Feynman, J., and N. V. Crooker, The solar wind at the turn of the century, Nature, 275, 626-627,1978.

Habbal, S. R., Variable EUV emission in the quiet Sun and coronal heating, in: Solar Wind Seven, Eds. E. Marsh, R. Schwenn, Pergamon Press Oxford, New York, Seoul, Tokyo, pp. 41-48, 1992.

Hoeksema, J. T., and P. H. Scherrer, The solar magnetic field-1976 through 1985: An atlas of photospheric magnetic field observations and computed coronal magnetic fields from the John M. Wilcox solar observatory at Stanford, 1976-1985, Rep. A UG94, World Data Center A for Solar-terrestrial physics, Boulder Colo., 1986.

Howard, R., Observations of solar magnetic fields, Astrophys. J., 130, 193-201., 1959.

Hoyt, D. V., K. H. Schatten, and E. Nesme-Ribes, The one hundredth year of Rudolf Wolf's death: Do we have the correct reconstruction of solar activity? Geophys. Res. Lett., 21, 20672070, 1994.

Kocharov, G. E., Nuclear processes in the solar atmosphere and the particle acceleration problem, Astrophys. Space Sci., 6, 155-202, 1987.

Kojima, M., and T. Kakinuma, Solar cycle dependence of global distribution of solar wind speed, Space Sci. Rev., 53, 173-222, 1990.

Krause, F., and H. Radler, Mean-Field Magnetohydrodynamics and Dynamo Theory, Pergamon Press, Oxford, 1980.

Legrand, J. P., and P. A. Simon, Some solar cycle phenomena related to the geomagnetic activity from 1868 to 1980 , I. The shock events of the interplanetary expansion of the toroidal field, Astron. Astrophys., 152, 199-204, 1985.

Legrand, J. P., and P. A. Simon, Shock waves, solar streams and the spread of aurorae in latitude, J. Brit. Astron. Assoc., 98, 311-312,1988.

Leighton, R.B., Observations of solar magnetic fields in plage regions, Astrophys. J., 130, 366-380, 1959.

Mendoza, B., Solar irradiance during the Maunder minimum, Geofis. Int., 35, 161-167, 1996.

Parker, E., Interplanetary Dynamical Processes, Interscience Publ. Wiley and Sons, New York-London, 1963.

Perez-Enríquez, R., and B. Mendoza, Response of the Earth to crossings of the heliospheric current sheet, J. Geophys. Res., 98 19349-19354, 1993.

Ribes, E., Ph. Merlin, J.-C. Ribes, and R. Barthalot. Absolute periodicities in the solar diameter, derived from historical and modern data, Ann. Geophysicae, 7, 321-330, 1989.

Ribes, J. C., and E. Nesme-Ribes, The solar sunspot cycle in the Maunder minimum AD 1645 to AD 1715, Astron. Astrophys., 276, 549-563, 1993.

Schroeder, W., On the existence of the 11-year cycle in solar and auroral activity before and during the so-called Maunder minimum, J. Geomagn. Geoelectr., 44, 119-128, 1992.

Silverman, S. M., Magnetic activity and solar wind velocity at and preceding solar minimum for the past 500 years, J. Geophys. Res., 91, 10157-10161, 1986.

Silverman, S. M., Secular variations of the Aurora for the past 500 years, Rev. Geophys., 30, 333-351, 1992.

Simon, P. A., and J. P. Legrand, Some solar cycle phenomena related to the geomagnetic activity from 1868 to 1980. II, Highvelocity wind streams and cyclical behavior of poloidal field, Astron. Astrophys., 155, 227-236, 1986.

Simon, P. A., and J. P. Legrand, Some solar cycle phenomena related to the geomagnetic activity from 1868 to 1980 . III, Quiet days, fluctuating activity or the equatorial belt as the main origin of the solar wind flowing in the ecliptic plane, Astron. Astrophys., 182, 329-336, 1987.

Simon, P. A., and J. P. Legrand, Solar cycle and geomagnetic activity: A review for geophysicists. Part II. The solar sources of geomagnetic activity and their links with sunspot cycle activity, Ann. Geophysicae, 7, 579-594, 1989.

Slavin, J. A., and E. J. Smith, Solar cycle variations in the interplanetary magnetic field, Solar Wind Five, NASA Conf. Publ. CP 2280, 323-331, 1983.

Suess, S. T., The solar wind during the Maunder minimum, Astrophys. Space Sci., 27, 1001-1013, 1979.

Wilson, O. C., Chromospheric variations in main sequence stars, Astrophys. J., 226, 379-396, 1978. 